Ann. Zootech., I965, 14 (4), 40i-408.

\title{
RÉSULTATS PRÉLIMINAIRES CONCERNANT L'INFLUENCE DU VERRAT SUR LA TAILLE DE LA PORTÉE EN INSÉMINATION ARTIFICIELLLE
}

\author{
C. LEGAULT et L. OLLIVIER \\ avec la collaboration technique de B. Dando et L. Guilbault \\ Station centrale de Génétique animale, Jouy-en-Josas (Seine-et-Oise) ('). \\ Centre national de Recherches zootechniques, \\ Centre expérimental de Sélection et d'Insémination porcine, Rouillé (Vienne).

\section{SOMMAIRE} \\ L'effet direct du verrat sur la taille des portées qu'il engendre a été estimé sur I I 39 portées \\ contrốlées à la naissance et 1022 portées contrôlées au sevrage. Ces portées sont issues de 19 verrats \\ de race Large White utilisés de 1961 à 1964 par le Centre expérimental de Sélection et d'Insémi- \\ nation' porcine de Rouillé (Vienne). L'analyse statistique met en évidence, un effet hautement \\ significatif du verrat sur la taille de la portée à la naissance. Cependant, l'effet du verrat n'est plus \\ significatif au sevrage, par suite, vraisemblablement, d'une mortalité plus élevée dans les grandes \\ portées qui atténue les différences observées à la naissance.
}

\section{I. - INTRODUCTION}

Les effectifs de verrats et de truies sont, en France, dans le rapport I à 7 pour les élevages de sélection (MOLENat et POUlenc, I962) et dans le rapport I à 23 pour l'ensemble du pays (ZER'T, I965). Dans ces conditions, l'effet que peut exercer un verrat sur la taille des portées qu'il engendre est généralement confondu avec l'effet " élevage " et par suite difficile à estimer. De plus, pour atteindre des nombres suffisants de portées par verrat, il est nécessaire de considérer des périodes assez longues, ce qui introduit des effets annuels et saisonniers d'une réelle importance.

(1) Ce travail a été partiellement financé par l'Association nationale de Contrôle des Performances de l'Espèce porcine, i 6 rue Claude Bernard, Paris $\left(5^{\mathrm{e}}\right)$. 
En revanche, dans le cadre d'un centre d'insémination artificielle, plusieurs. verrats, dont certains engendrent plus de mille portées par an, peuvent être mis. en comparaison; dans de telles conditions, l'effet qu'ils ont sur la taille de la portée, s'il existe, cesse d'être négligeable.

De nombrewx auteurs affirment que, du moment qu'il est fertile et entretenu dans de bonnes conditions, le verrat n'a aucune influence sur la taille des portées. qu'il engendre: KRALIINGER et SChOTT (I934), DzAPARIDZE (I935), Musson (I946), REDDY et coll. (I958) et WILSON et coll. ( 1962 ). Cependant, d'autres études démontrent l'influence significative du verrat sur la taille de la portée, soit en monte naturelle : Barbosa (I962), SkJERVOLI) (I963), soit en insémination artificielle: Van Oers (I964) et Maijala (1965). Ces derniers résultats sont d'ailleurs en accord avec ceux obtenus par FALCONER (I960) et Finn (I964) sur la souris.

L'objectif de cette étude est précisément d'estimer l'effet direct des verrats utilisés en insémination artificielle sur la taille de la portée à la naissance et au sevrage.

\section{II. - MA'TÉRIEI, ANIMAI, ET MÉTHODF D'ANALYSE}

Les données proviennent d'un ensemble de I 500 portées de la race Large White, soumises chacune à deux contrôles dans l'aire d'extension du Centre expérimental de Sélection et d'Insémination porcine de Rouillé (Vienne) de 1961 à 1964 . Les contrôles, effectués par des techniciens de l'I. N. R. A., consistaient à dénombrer et à peser les portées, dans les quatre jours qui suivent la naissance pour le premier contrôle, entre les âges de 48 et $5^{8}$ jours pour le second.

Dans la présente étude, nous n'avons retenu que les 19 verrats qui avaient un minimum de 33 portées contrôlées à la naissance, ce qui constitue un échantillon de I I 39 portées au premier contrôle et réduit à I 022 portées au second contrôle. Au cours de la même période, 15 autres verrats avaient été éliminés après avoir engendré un petit nombre de portées, soit par suite d'accident ou pour des raisons sanitaires, soit à cause d'un pourcentage de réussite insuffisant (inférieur de so à I2 points à la moyenne contemporaine).

Trois variables ont été étudiées :

- variable I : le nombre total de porcelets nes (vivants et morts) indiqué généralement par l'éleveur au moment du premier contrôle;

- variable 2 : le nombre de porcelets vivants a la première pesée;

- variable 3 : le nombre de porcelets vivants a la seconde pesée.

Nous avons analysé l'effet du verrat ( 9 niveaux), l'effet de l'année de mise-bas (4 niveaux) et l'effet du numéro de portée ( 3 niveaux : première, seconde, troisième portée et portées d'ordre plus élevé). I.es effets attachés à chacun des niveaux des trois facteurs ont été estimés par la méthode des moindres carrés, une fois le test de l'hypothèse de non-interaction réalisé. Tous les calculs ont été réalisés sur ordinateur I. B. M. $1620{ }^{(1)}$ selon la méthode de STEvens (I951). La taille de la portée a été représentée par le modèle mathématique suivant :

$$
y_{i j k l}=\mu+v_{l}+a_{j}+o_{k}+s_{l j k l}
$$

dans lequel :

$-y_{i j k l}$ est la taille d'une portée;

- $\mu$ est la moyenne générale;

-. $v_{i}$ représente l'effet du père de cette portée avec $i=1,2 \ldots . .19$;

- $a_{j}$ représente l'effet de l'année dans laquelle est née cette portée avec : $j=\mathbf{1 , 2 , 3 , 4}$;

-- ok représente l'effet du numéro d'ordre de la portée avec $k=\mathbf{I}, \mathbf{2}, \mathbf{3}$;

- $s_{i j k l}$ représente l'erreur aléatoire de moyenne nulle et de variance $\sigma_{e}^{2}$

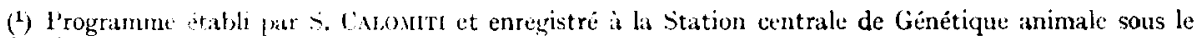
numéro $6402_{\%}$. 


\section{III. - RÉSULTATS}

Le tableau I donne les résultats des analyses de la variance aboutissant aux tests des interactions.

\section{TABLEAU I}

Tests des interactions

\begin{tabular}{|c|c|c|c|c|c|c|c|c|}
\hline \multirow{2}{*}{$\begin{array}{l}\text { Source } \\
\text { de variation }\end{array}$} & \multicolumn{3}{|c|}{ Variable $1(\mathbf{1})$} & \multicolumn{2}{|c|}{ Variable $2\left({ }^{2}\right)$} & \multicolumn{3}{|c|}{ Variable 3 ( $(2)$} \\
\hline & $\begin{array}{l}\text { degrés } \\
\text { de liberté }\end{array}$ & $\begin{array}{l}\text { carrés } \\
\text { moyens }\end{array}$ & $\mathrm{F}$ & $\begin{array}{c}\text { carrés } \\
\text { moyens }\end{array}$ & $\mathrm{F}$ & $\begin{array}{c}\text { degrés } \\
\text { de liberté }\end{array}$ & $\begin{array}{l}\text { carrés } \\
\text { moyens }\end{array}$ & $\mathbf{F}$ \\
\hline Effets principaux & 23 & 85,26 & & 44,72 & & 23 & 26,89 & \\
\hline Interactions .... & 104 & 12,36 & $0,98 \mathrm{NS}$ & 7,05 & $0,78 \mathrm{NS}$ & 101 & 6,82 & $0,83 \mathrm{NS}$ \\
\hline Entre cellules.... & 127 & 25,56 & & 13,87 & & 124 & 10,54 & \\
\hline $\begin{array}{r}\text { Résiduelle (intra- } \\
\text { cellules) } \ldots \ldots\end{array}$ & 1011 & 12,57 & & 9,04 & & 897 & 8,15 & \\
\hline Totale & 1138 & & & & & 1021 & & \\
\hline
\end{tabular}

(1) Nombre de porcelets nés par portée.

(2) Nombre de torcelets vivants par portée au premier contrôle.

$\left({ }^{9}\right)$ Nombre de porcelets vivants par portée au second contrôle.

Le tableau 2 donne les résultats des analyses de la variance aboutissant aux tests des effets du verrat, de l'année de mise-bas, et du numéro de portée dans l'hypothèse de nullité de 1'interaction entre ces trois facteurs.

Dans le tableau 3 figurent les effectifs et les effets relatifs à chacun des niveaux des trois facteurs étudiés.

Les résultats peuvent se résumer de la manière suivante :

I. L'examen du tableau I montre que les interactions entre les facteurs de variation ne sont significatives pour aucune des trois variables. Il est à noter que le test concerne globalement trois interactions de $I^{\text {er }}$ ordre et une interaction de $2^{\mathbf{e}}$ ordre. Par ailleurs, les trois tests des interactions entre les facteurs pris deux à deux ont été effectués en supposant le troisième facteur indépendant, et, dans tous les cas, ils ont confirmé l'absence d'interaction.

2. L'effet du numéro de portée est hautement significatif, que ce soit à la naissance ou au sevrage, et il est particulièrement sensible entre la première portée et les portées ultérieures. Ce résultat confirme d'ailleurs de nombreuses observations antérieures.

3. L'année de mise bas n'a pas d'effet significatif sur la taille de la portée. Il est cependant probable que des effets saisonniers que nous n'avons pas estimés existent réellement. 
4. Ėnfin, l'effet direct du verrat sur la taille de la portée est hautement significatif à la naissance, significatif au premier contrôle, et n'est plus significatif au sevrage.

\section{TABLEAU 2}

Tests des effets du verrat, de l'année de mise bas, et du numéro de portée dans l'hypothèse de non-interaction entre les 3 facteurs

\begin{tabular}{|c|c|c|c|c|c|c|c|c|}
\hline \multirow{2}{*}{$\begin{array}{c}\text { Source } \\
\text { de variation }\end{array}$} & \multicolumn{3}{|c|}{ Variable $1\left({ }^{1}\right)$} & \multicolumn{2}{|c|}{ Variable $2\left({ }^{2}\right)$} & \multicolumn{3}{|c|}{ Variable $3\left({ }^{3}\right)$} \\
\hline & $\begin{array}{l}\text { degrés } \\
\text { de liberté }\end{array}$ & $\begin{array}{l}\text { carrés } \\
\text { moyens }\end{array}$ & $\mathrm{F}$ & $\begin{array}{l}\text { carrés } \\
\text { moyens }\end{array}$ & $\mathrm{F}$ & $\begin{array}{l}\text { degrés } \\
\text { de liberté }\end{array}$ & $\begin{array}{l}\text { carrés - } \\
\text { moyens }\end{array}$ & $\mathrm{F}$ \\
\hline Verrat ......... & 18 & 33,18 & $2,64^{* *}$ & 15,08 & $1,70^{*}$ & 18 & 10,50 & $1,30 \mathrm{NS}$ \\
\hline Année...$\ldots \ldots$ & 3 & $\quad 13,46$ & $1,07 \mathrm{NS}$ & ' $4 ; 34$ & $0 ; \% 9 \cdot \mathrm{NS}$ & 3 & 4,04 & $-0,50 \mathrm{NS}$ \\
\hline No de portée... & 2 & 467,40 & $37,22^{* *}$ & 317,11 & $35,82 * *$ & 2 & 176,70 & $22 ; 03^{* * *}$ \\
\hline Résiduelle.. & 1011 & 12,57 & & 9,04 & & 998 & $8 ; 15$ & $\begin{array}{l}\cdots \\
\therefore,\end{array}$ \\
\hline
\end{tabular}

NS : Non significatif au seuil de 5 p. 100.

* : Significatif au seuil de 5 p. 100.

** : Significatif au seuil de 1 p. 100.

(1) Nombre de porcelets nés par portée.

(2) Nombre de porcelets vivants par portée au premier contrốle.

(3) Nombre de porcelets vivants par portée au second contrôle.

\section{IV. - DISCUSSION}

Ce dernier résultat est à vrai dire le seul qui nous initéresse dans cette étude aussi réclame-t-il une prudente discussion.

En premier lieu, nous devons souligner l'importance de l'effet direct du verrat révélée par l'examen du tableau 3. La différence entre le verrat le plus prolifique et le verrat le moins prolifique est en effet égale à 2,56 porcelets par portée à la naissance, 2,09 au premier contrôle et 2,2 I au sevrage.

En second lieu, rappelons que l'échantillon des verrats étudiés n'est pas représentatif de la population totale, une sélection sévère ayant eu lieu. E,n effet, les verrats retenus ont un grand nombre de portées contrôlées; cela signifie qu'ils ont été utilisés pleinement dans le centre d'insémination artificielle. Par contre, les verrats ayant un pourcentage de réussite insuffisant ou produisant un sperme de mauvaise qualité ont été éliminés après avoir engendré un petit nombre de portées. A titre indicatif, nous avons estimé la taille moyenne, aux trois périodes de référence, de 95 portées issues des 15 verrats éliminés prématurément. Les valeurs trouvées, égales respectivement à $8, \operatorname{II}_{5}, 7,202$ et 7,06 sont toutes inférieures aux moyennes 
générales de l'échantillon retenu pour cette étude (qui sont respectivement : ro,049 8,760 et 8,026 porcelets par portée).

DU Mesnil du Buisson et Signoret (communications personnelles) et PAREdis (Ig62) ont d'ailleurs remarqué l'existence d'une relation entre le pourcentage de

\section{TABLEAU 3}

Effets du verrat, de l'année, et du numéro de portée sur la taille de la portée estimés par la méthode des moindres-carrés

\begin{tabular}{|c|c|c|c|c|c|c|}
\hline $\begin{array}{c}\text { Facteur } \\
\text { de variation }\end{array}$ & Niveau & $\begin{array}{l}\text { Nombre } \\
\text { de portées }\end{array}$ & Variable $1{ }^{(1)}$ & Variable $2\left({ }^{2}\right)$ & $\begin{array}{l}\text { Nombre } \\
\text { de portées }\end{array}$ & Variable $3\left(^{3}\right)$ \\
\hline $\begin{array}{c}\text { Verrat } \\
\text { (numéro) }\end{array}$ & $\begin{array}{l}0230 \\
0262 \\
0301 \\
0302 \\
0303 \\
0316 \\
0568 \\
0712 \\
0722 \\
0977 \\
5255 \\
5294 \\
5455 \\
5627 \\
5648 \\
5698 \\
6003 \\
6355 \\
9043\end{array}$ & $\begin{array}{r}65 \\
53 \\
67 \\
65 \\
67 \\
62 \\
34 \\
74 \\
36 \\
67 \\
33 \\
139 \\
43 \\
51 \\
38 \\
63 \\
33 \\
71 \\
78\end{array}$ & 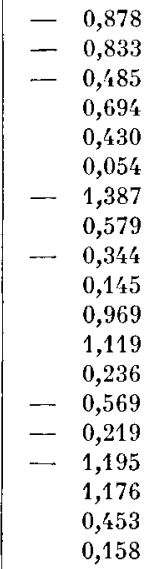 & $\begin{array}{r}-0,356 \\
-0,635 \\
-0,557 \\
0,211 \\
0,115 \\
0,122 \\
-0,672 \\
0,376 \\
0,270 \\
0,205 \\
0,983 \\
0,719 \\
-0,092 \\
-0,541 \\
0,042 \\
-1,113 \\
0,973 \\
-0,723 \\
-0,009\end{array}$ & $\begin{array}{r}61 \\
49 \\
60 \\
62 \\
58 \\
53 \\
30 \\
65 \\
30 \\
66 \\
29 \\
125 \\
33 \\
45 \\
36 \\
55 \\
28 \\
64 \\
73\end{array}$ & $\begin{array}{r}0,355 \\
-0,862 \\
-0,375 \\
0,132 \\
0,283 \\
0,254 \\
-0,273 \\
-0,105 \\
0,440 \\
0,149 \\
0,995 \\
0,179 \\
0,221 \\
-0,401 \\
0,238 \\
-1,108 \\
1,101 \\
-0,202 \\
-0,246\end{array}$ \\
\hline Année & $\begin{array}{l}1961 \\
1962 \\
1963 \\
1964\end{array}$ & $\begin{array}{l}285 \\
270 \\
374 \\
260\end{array}$ & $\begin{array}{r}0,007 \\
-\quad 0,249 \\
0,324 \\
-\quad 0,082\end{array}$ & $\begin{array}{r}-0,027 \\
-0,153 \\
0,195 \\
-0,015\end{array}$ & $\begin{array}{l}255 \\
250 \\
299 \\
218\end{array}$ & $\begin{array}{r}-0,061 \\
-0,123 \\
0,201 \\
-0,017\end{array}$ \\
\hline $\begin{array}{c}\text { Numéro } \\
\text { de portée }\end{array}$ & $\begin{array}{l}1 \\
2 \\
3 \text { et }+\end{array}$ & $\begin{array}{l}276 \\
191 \\
672\end{array}$ & $\begin{array}{r}1,123 \\
0,237 \\
0,992\end{array}$ & $\begin{array}{r}-1,114 \\
0,465 \\
0,671\end{array}$ & $\begin{array}{l}238 \\
172 \\
612\end{array}$ & $\begin{array}{r}0,942 \\
0,469 \\
0,473\end{array}$ \\
\hline Moyenne & & & 10,049 & 8,670 & & 8,026 \\
\hline Effectif total & & 1139 & & & 1022 & \\
\hline
\end{tabular}

(1) Nombre de porcelets nés par portée.

$\left({ }^{2}\right)$ Nombre de porcelets vivants par portée au premier contrôle.

$\left({ }^{3}\right)$ Nombre de porcelets vivants par portée au second contrôle.

réussite après insémination première et la taille de la portée à la naissance : cette observation a été confirmée par van OERs (1964) et MAIJALA (I965) qui ont trouvé des corrélations positives voisines de 0,50 entre ces deux variables.

Ces remarques laissent donc supposer que l'effet réel du verrat est plus impor-

Annales de Zootechnie. - I965. 
tant que celui que nous avons estimé. Cependant, il faut admettre que notre étude ne concerne que des verrats qui remplissent les conditions d'une utilisation "normale " par un C. I. A.

Le fait que l'effet du verrat sur la taille de la portée cesse d'être significatif au sevrage s'explique probablement par une mortalité plus élevée dans les grandes portées, ce qui atténue les différences entre verrats.

Le mâle peut intervenir sur la prolificité de deux manières, soit par son influence sur le nombre d'ovules fécondés, soit par son influence sur le nombre d'embryons se développant normalement jusqu'à la naissance. I1 a cependant été démontré que 87 p. Ioo à 96 p. Ioo des ovules sont fertilisés : RoBERTson et coll. (I95 I), HANI, (I96r), PERRY et Rowlands (I962). C'est donc avant tout sur la mortalité embryonnaire que le mâle peut avoir une action sensible.

Bishop (I964) souligne le fait que la mortalité embryonnaire est due en grande partie à des causes génétiques, le mâle intervenant par l'intermédiaire de facteurs léthaux qui lui sont transmis par ses ascendants. KRITSJANSSON (I964) a constaté que l'un des 9 types de croisement possibles à partir des 3 génotypes correspondant à un locus de $\beta$-globulines est caractérisé par un fort pourcentage de retours en chaleur qu'il attribue à une mortalité embryonnaire accrue. Une observation analogue a été faite par AshToN (I96r) sur les bovins. Finfin, nous pouvons citer la découverte récente d'un verrat porteur d'une translocation hétérozygote dont la prolificité était réduite de moitié (BENGT et LENNART, I964).

Bishop insiste sur l'importance longtemps méconnue des mutations qui appa raissent spontanément ou à la suite de l'action d'agents mutagènes sur le spermatozoïde, soit au cours de sa formation, soit au cours de sa conservation in vitro ("vieillissement du sperme "). Une baisse de la taille de la portée survenant à la suite de l'irradiation des testicules, observée par de nombreux auteurs chez la souris et le cobaye et par Cox et Wri,LHAM (Ig6z) chez le porc, confirme cette hypothèse.

Du point de vue de l'amélioration d'une population, la possibilité d'effectuer une sélection des verrats sur la taille des portées qu'ils engendrent a été envisagée par SKJERVOLD (1962). A ce sujet, il faut remarquer qu'une telle sélection se fait inévitablement, mais il reste à prouver dans quelle mesure une amélioration ainsi obtenue se transmet d'une génération à la suivante.

\section{VI. - CONCIUSION}

Le résultat intéressant de cette étude est la mise en évidence d'une influence marquée du verrat " normalement prolifique "sur le nombre de porcelets mis bas, cette influence devenant de moins en moins nette à mesure que l'on se rapproche du sevrage.

Sur le plan pratique, nous pouvons retenir qu'un effet dépressif du verrat sur la taille de la portée, même faible, peut se traduire dans un C. I. A. par un déficit annuel supérieur à mille porcelets. Lans les conditions de milieu de notre échantillon, 
les pertes entre la naissance et le sevrage atténuent ces différences; il est donc vraisemblable que l'élimination des verrats les moins prolifiques n'aurait d'effet que dans les élevages où la mortalité dans le jeune âge est minimum.

Rę̧u pour publication en juillet 1965 .

\section{SUMMARY}

INFLUENCE OF TIIE BOAR ON LITTER SIZE IN ARTIFICIAL INSEMINATION

The effect of the boar on litter size has been estimated from a sample of 1 I 39 litters at birth and 1022 litters at weaning. Those litters were sired by 19 Large White bours from i961 to 1964 at an experimental $A$. I. Center located at Rouillé (Vienne). The effects of the boar, the year of birth and the litter order were estimated by least squares. The results may be summarized in the following way :

- there was no significant interaction between boar, year and litter order ;

- the effect of the litter order was highly significant both at birth and at weaning;

- the effect of the year was not significant.

- the effect of the boar on litter size was highly significant at birth, but not significant at weaning, probably owing to a higher mortality in large litters. However, the sample of boar stu. died was partly selected on farrowing rate and this may have resulted in an underestimation of the boar effect.

\section{RÉTÉRENCES BIBLIOGRAPHIQUES}

Asirton G. C., ig6r. B-Globulin type and fertility in artificially bred dairy cattle. J.Reprod.Fertil,, 2, i1 7129 .

Barbosa A. S., 1962. Some factors affecting litter size and weight and pigling variability at go days in Berkshire and Duroc-Jersey pigs bred at the "Diaulas Abreu "College of Agricultural Technology. Arq. Esc. Veter. Univ. Minas. Gerais, 13, 255-29r (in Anim. Breed. Abstr. 32, г 204).

Bengt H., Lenvart B., I964. Translocation heterozygosity in a boar. Ilevedilas, 52, I66-r 70 .

Brsnop M. W. II., 1964. Paternal contribution to embryonic death. J. Reprod. Fertil., 7, 383-396.

Cox D. F., Willaha R. I., 1962. Genetic effects of irradiation on early mortality in swine. Genetics, $47,785-788$.

DZAPARIDZE., r935. Studies on the estimution of fertility and weight at birth and at four weeks in the IJannover-Braunschweig land pir. Zilchtungshunde, 10, 208-217 (in Anim. Breed. Abstr., 4, 203).

Falconer D. S., 1960. The genetics of litter size in mice. J. Cell and Comp. Physiol., 58, r53-167.

Fine C. A., 1964 . Influence of the male on litter size in mice. J. Reprod. Ficrili., 7, ro7-ris.

Hanly S., igGr. I'renatal mortality in farm animals. J. Reprol. Fertil., 2, isz-194.

Krallinger II. F., Schotr A., 1934. The influence of the boar on litter size. Anim. Rreed. Absir. 2,127 .

Kritsjansson Ti. K., ig64. Transferrin types and reproductive performance in the pir. J. Reprod. Fertil., 8, 3 II -318.

Maijala K., 1965. Communication aux Journées d'Études de la F. E. Z. Noordwijk. Commission de Génétique.

Messil du Buisson F. du, Signoret J. P'., 1965. Communications personnelles.

Molevat M., Poulenc J., 1962. Performances d'étevare et séluction du porc Large White. Bull. Tech. Ing. Sery. Agric., 171, 617-684.

Musson A. L., 1964 . The influence of the boar on litter size. J. Anim. Sci, $5,418$.

OERs J. P. S. van, 1964. De invlozd van de beer op de worpgrootte. Veeteelt en Zuivelberichten, 6, 5 I I-520.

Paredis F., I962. Fertility and artificial insemination in pigs. Intern. J. Ficrili., 7, 223-233.

Perry J. S., Rowlands I. W., rg62. Tiarly pregnancy in the pig. J. Reprod. Fertil., 4, I 75- 88.

Innales de Zootechnie. - r965. 
ReduY V. B., LASLEY J. H., MIAYER D. I., I958. Crenetic aspects of reproduction in swine. Unizersity of Missotiri Res. Bull. $\mathbf{6 6 8}$.

Robertson G. L., Casida I. E., Grummer R. H., Chapmaí A. B., 195i. Some feeding and management factors affecting age at puberty and related phenomena in Chester White and Poland-China gilts. J. Anim. Sci., 10, $841-856$.

SkJervoli, H., I963. To what extent do boar affect the litter size. Meld. Norges Landbrukshogsk., 42, I I Pp.

STevens W. I., I95I. Asymototic regression. Biometrics, 7, $247-267$.

Wilson S. P., Wilatley J. A. Jr., Whiteman J. V., Morrison R. I)., ig62. Influence of sire and line of breeding on sow productivity. J. Anim. Sci., 21 , $119-122$.

ZERT P., 1965. Le porc dans la Communauté Économique Huropécnne. Le Porc, 36, $15-48$. 